\title{
Deuteron cross section evaluation for safety and radioprotection calculations of IFMIF/EVEDA accelerator prototype
}

\author{
Valentin Blideanu ${ }^{\text {a }}$, Mauricio García ${ }^{\text {b.c }}$, Philippe Joyer ${ }^{\mathrm{a}, *}$, Daniel López ${ }^{\mathrm{b}, c}$, Alicia Mayoral ${ }^{\mathrm{b}, c}$, \\ Francisco Ogando ${ }^{b . c}$, Félix Ortíz ${ }^{b}$, Javier Sanz ${ }^{b . c}$, Patrick Sauvan ${ }^{b . c}$ \\ a Commissariat à l'énergie atomigue CEA/IRFU, Centre de Saday, 91191 Gif sur Yvette cedex, France \\ 'Universidad Nacional de Educación a Distancia, UNED, Madrid, Spain \\ "Instituto de Fusión Nuclear, UPM, Madrid, Spain
}

\begin{abstract}
A B S T R A C T
In the frame of IFMIF/EVEDA activities, a prototype accelerator delivering a high power deuteron beam is under construction in Japan. Interaction of these deuterons with matter will generate high levels of neutrons and induced activation, whose predicted yields depend strongly on the models used to calculate the different cross sections. A benchmark test was performed to validate these data for deuteron energies up to $20 \mathrm{MeV}$ and to define a reasonable methodology for calculating the cross sections needed for EVEDA. Calculations were performed using the nuclear models included in MCNPX and PHITS, and the dedicated nuclear model code TALYS. Although the results obtained using TALYS (global parameters) or Monte Carlo codes disagree with experimental values, a solution is proposed to compute cross sections that are a good fit to experimental data. A consistent computational procedure is also suggested to improve both transport simulations/prompt dose and activation/residual dose calculations required for EVEDA.
\end{abstract}

\section{Introduction}

Engineering validation and engineering design activities (EVEDA) are the first phase of the JFMIF project and began in mid 2007 [1]. Engineering activities include the three main parts of the future IFMIF facility: the accelerator, the target and the test facilities. A prototype of the IFMIF accelerator is under construction in Japan at the Rokkasho-Mura site. The accelerator sub-systems and components will be provided by the European team that is in charge of safety and radioprotection calculations for this equipment. The prototype accelerator will deliver a high power deuteron beam of $125 \mathrm{~mA}$ at $9 \mathrm{MeV}$ that is lower than IFMIF, which will use two deuteron beam-lines each delivering $125 \mathrm{~mA}$ at $40 \mathrm{MeV}$.

In the accelerator prototype, these deuterons will interact with materials:

- along the beam line wherever deuterons are lost,

- at the end of the beam line, in the beam dump where the whole beam is stopped.

Depending on the energy of the deuterons, their interaction with matter will then produce high neutron levels as well as induced activation. The production of neutrons and radioactive inventories due to deuteron-induced reactions is a major issue for radioprotection and safety studies in EVEDA and in IFMIF.

The Monte Carlo codes MCNPX [2] and PHITS [3] are widely used in accelerator radioprotection studies. They use built-in analytical models to deal with deuteron nuclear interactions. Although these models are designed to deal with high energy charged particles, the response of them, if used for the EVEDA low energy range, is worth being evaluated. The first objective of this work was to test these codes regarding their capability to predict neutron production for EVEDA conditions, as well as to predict nuclear reaction rates producing radioactive nuclides.

In some EVEDA radioprotection studies the applicability of MCNPX to predict neutron production with sufficient accuracy was found to be questionable. In this respect a new computationa] procedure was proposed [4] based on extending MCNPX to use data files for neutron (and gamma) production for deuteroninduced reactions generated by the TALYS code [5]. In this context, the second objective of this work was to test the capability of TALYS to generate these data files for neutron production and for deuteron induced activation cross sections with adequate accuracy. Discussion of the available deuteron activation data file EAF-2007 [6] is also included.

Using a good set of input nuclear data and evaluating their accuracy are preliminary necessary tasks required to improve the safety and radjoprotection calculations needed for the JFMIF/ 
EVEDA phase. A benchmark was proposed between the two European home teams involved in safety and radioprotection calculations for IFMIF/EVEDA (UNED and CEA) in order to define the best methodology to calculate and make use of the deuteron cross sections needed for EVEDA calculations.

\section{Description and objectives of the benchmark}

The main goal of the proposed benchmark is to compare calculated cross sections using different computer codes used for safety and radioprotection calculations with experimental data available for different elements relevant to the JFMIF/EVEDA project. These elements are: $\mathrm{Cu}, \mathrm{Ni}, \mathrm{Fe}, \mathrm{W}, \mathrm{C}, \mathrm{Cr}$, and $\mathrm{Nb}$ and are typical of the materials used in components of accelerators ( $\mathrm{Cu}$ for the beam dump, stainless steel for the vacuum chamber, $\mathrm{Nb}$ and $\mathrm{W}$ for other specific equipment such as the superconducting drift tube linac). The present benchmark has focused on the EVEDA phase and the energy has been set in the range $0-20 \mathrm{MeV}$.

The benchmark has been performed in three steps:

- In the first step, an inventory of the available experimental data in EXFOR regarding the excitation functions of the deuteron-induced reactions for these elements has been compiled.

- In the second step, the different cross sections has been calculated with the different nuclear models included in MCNPX and PHITS.

- In the third step, the different cross sections have been calculated with the dedicated nuclear reaction code TALYS.

Dominant nuclear reactions were identified and their cross sections validated by comparing the calculated and available experimental values. The results obtained are discussed in the following sections for neutron production and for induced activation.

\section{Benchmark analysis for neutron source generation}

Cross sections calculated with MCNPX-2.6 (using INCL4/ABLA and ISABEL/Dresner/RAL model options) and PHITS (QMD model) show that for the majority of the reactions there is a very strong disagreement in both energy dependence profile and values. For the other nuclear models included in MCMPX (such as CEMO3 and LAQGSM) the situation is even worse. Thus, it turned out that the present versions of the nuclear models included in MCNPX and PHITS are not appropriate for dealing with deuteron-induced reactions in the energy range of EVEDA applications.

TALYS- 1.0 cross sections obtained using the default-global set of optical model potential (OMP) parameters reproduce well the energy dependence of the experiments for the majority of the reactions. In quite a few cases this profile is quantitatively well adjusted to the experiments, while in some of them an existing quantitative disagreement is observed, and some fit to experimental values is required. Figs. 1 and 2 are examples of reactions (for which recent experimental data are avajlable) showing the poor behavior of MCNPX and PHITS nuclear models, and the good agreement of TALYS- 1.0 cross sections with the experimental results [7]. The TALYS- 1.0 results in Fig. 1 were obtained using the default-global set parameters while in Fig. 2 results from calculations using local parameters specified by $M$. Avrigeanu (NIPNE) as part of an EFDA Task [8] are also presented. The results obtained for the reaction ${ }^{65} \mathrm{Cu}(\mathrm{d}, 2 \mathrm{n}){ }^{65} \mathrm{Zn}$ using this local parameter set show a reasonable overestimate of experimental values [9-11]. Fig. 2 also shows that a best fit is possible by adjusting values between the two sets of parameters.

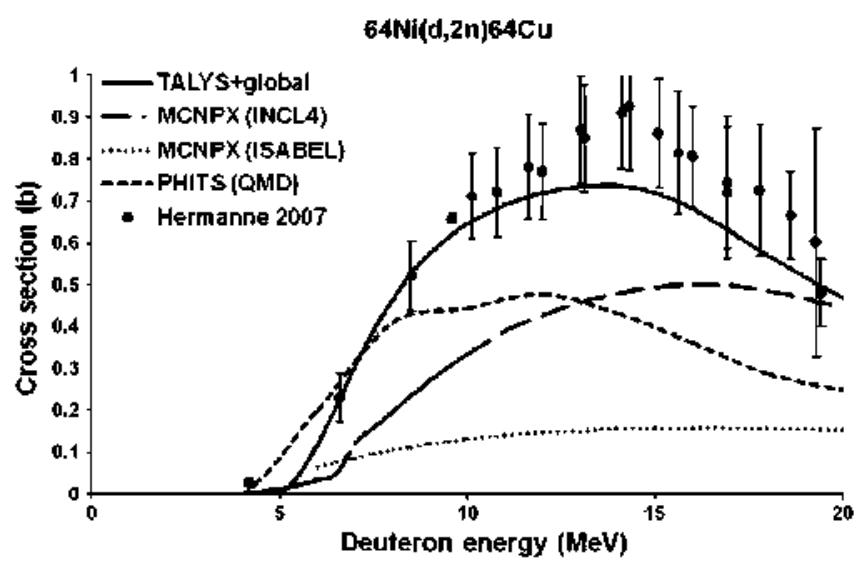

Fig. 1. Cross section for ${ }^{64} \mathrm{Ni}\left(d_{2} 2 n\right)^{64} \mathrm{Cu}$ reaction: comparison of evaluated and measured data [8].

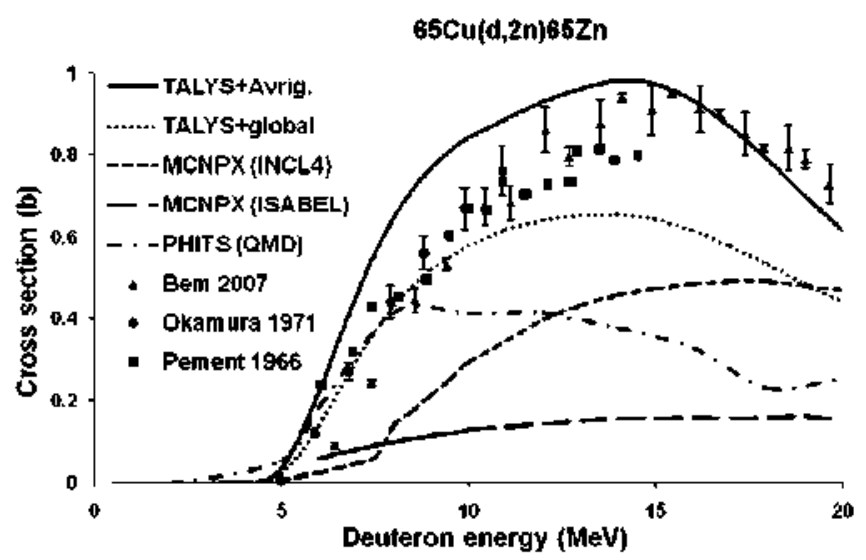

Fig. 2. Cross section for ${ }^{65} \mathrm{Cu}(\mathrm{d}, 2 \mathrm{n})^{65} \mathrm{Zn}$ reaction: comparison of evaluated and measured data [9-11].

Reactions such as ${ }^{61} \mathrm{Ni}(\mathrm{d}, \mathrm{n})^{62} \mathrm{Cu},{ }^{56} \mathrm{Fe}(\mathrm{d}, \mathrm{n})^{57} \mathrm{Co},{ }^{56} \mathrm{Fe}(\mathrm{d}, 2 \mathrm{n})^{56} \mathrm{Co}$ and ${ }^{56} \mathrm{Fe}(\mathrm{d}, \alpha){ }^{54} \mathrm{Mn}$ are some examples of reactions for which a qualitatively acceptable profile of the excitation function is obtained with TALYS-1.0 global parameters, but for which improvement in the fit to measurements is required. For these problematic cases TALYS-1.0 offers the capability of adjusting the parameters of the optical model potential involved in the interactions between the incident particle and the nucleus. To show that these kinds of problems can be solved reasonably, we have chosen the case of ${ }^{56} \mathrm{Fe}$. For this nuclide, experimental values for the excitation functions of three different reactions can be found in EXFOR data base and the results obtained with TALYS-1.0 (using defaultglobal parameters) reproduce well the energy dependence of the cross section defined by experimental data; however the quantitative fit is not good. We have chosen this case because for other nuclides in EXFOR there are values only for one (such as the case of ${ }^{61} \mathrm{Ni}(\mathrm{d}, n)^{62} \mathrm{Cu}$ ) or two reactions, and the fitting would be easier. Fitting calculations were done for all the experimental values and as a result a common set of parameters for the three reactions have been derived. Fitting calculations were done in this case by using only standard mathematical tools and all the experimental values given for the tree mentioned reactions. As the solution to the fitting problem, a common set of parameters for the three reactions were derived. Fig. 3 shows the improvement achieved with experimental values [12] for the reaction ${ }^{56} \mathrm{Fe}(\mathrm{d}, n){ }^{57} \mathrm{Co}$ using TALYS- 1.0 with the fitting parameter set over the TALYS- 1.0 results using the global parameters. With the selected parameter set an 


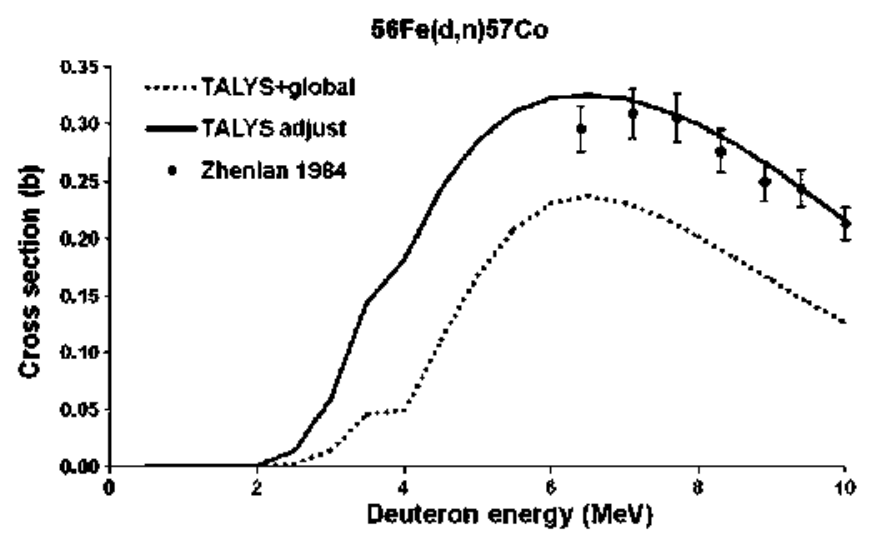

Fig. 3. Improved cross section for reaction ${ }^{56} \mathrm{Fe}(d, n)^{57} \mathrm{Co}$ using TALYS-1.0 [12].

improvement of ${ }^{56} \mathrm{Fe}(\mathrm{d}, 2 \mathrm{n}){ }^{56} \mathrm{Co}$ and ${ }^{56} \mathrm{Fe}(\mathrm{d}, \alpha)^{54} \mathrm{Mn}$ reactions - of interest for activation analysis - has also been achieved (as discussed in Section 4).

It is worth making some observations regarding some nuclides involved in this benchmark, such a Cu-63: (i) for them, the available experimental data in EXFOR are limited to a few reactions (only one in several cases) leading to the production of neutrons, (ii) TALYS- 1.0 reproduces adequately these data, (iii) the contribution of these reactions to the total production cross section as evaluated with TALYS-1.0 is very low (no experimental data for the total production cross section are available in EXFOR). Thus, for these particular nuclides, even though TALYS- 1.0 reproduces well the available experimental data, more effort on reliable prediction of the total neutron production cross section can be helpful. An example of these situations is shown in Fig. 4 for neutron production in ${ }^{63} \mathrm{Cu}$. This figure shows the calculated (with TALYS-1.0 and $M$. Avrigeanu parameters set) total neutron production cross section and its breakdown into the different contributing reactions. It reveals that the dominant reactions are $(d, n)$ and $(d, n p)$, and it is important to stress that no experimental data are available for these reactions: Therefore, an effort should be made to validate the TALYS results in computing the total neutron production cross section.

An estimation of neutron yield for $9 \mathrm{MeV}$ incident deuterons (n) d) on thick Cu- and Fe targets was performed using MCNPX models (ISABEL/Dresner/RAL and INCL4/ABLA) and TALYS-1.0. Calculations were also performed with PHITS for Cu. These results help to quantify the effect of the differences in the total neutron production cross sections up to $9 \mathrm{MeV}$ for MCNPX-2.6 models, PHTS and

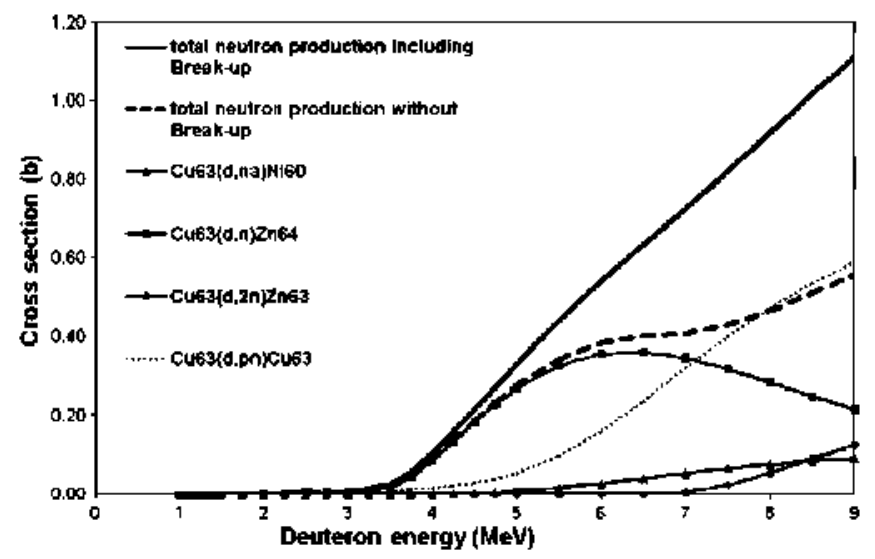

Fig. 4. Total neutron production cross section and breakdown contribution for ${ }^{63} \mathrm{Cu}$ (TAlYS-1.0 using M. Avrigeanu parameter's).
TALYS. Regarding TALYS-1.0 calculations, M. Avrigeanu optical model parameters and default values were used for $\mathrm{Cu}$ and $\mathrm{Fe}$, respectively. The results obtained are provided in Table 1 . This shows that MCNPX (with the INCL4/ABLA nuclear model that exhibits the better behavior) predicts a $\sim 3$ and a $\sim 30$ times lower yield than TALYS for natural $\mathrm{Cu}$ and Fe, respectively. For PHITS results, the agreement is slightly better, although the PHITS values are systematically below the TALYS results. Integral experiments of neutron yield for incident deuterons results necessary to assess the quality of these results, extending the available energy range.

other critical issue in regards to neutron production is the emitted spectrum. It is worth mentioning that the value computed with MCNPX-2.6 is not realistic at all because a nonphysical high energy tail is predicted, while TALYS-1.0 predictions are always consistent with the kinematics of the reactions. An example of this is provided in Fig. 5, which shows the angular integrated distribution for $9 \mathrm{MeV}$ deuterons incident on natural $\mathrm{Cu}$ computed by MCNPX2.6 (INCL4/ABLA), PHITS and TALYS-1.0 (global parameters) The maximum physical energy of the emitted neutrons is about 15.7 MeV. Nevertheless, more effort is needed to evaluate if the spectrum profiles provided by TALYS are accurate enough.

\section{Benchmark analysis for deuteron induced activation source generation}

Radionuclides produced by deuteron induced activation have been identified for all the elements in the benchmark as well as identifying the reactions dominating their production: $(d, \alpha)$, (d,p), (d,2p) and also $(d, x n)$ reactions. Similar comments to those reported in Section 3 regarding excitation functions for reactions contributing to neutron production can be made when comparing the corresponding results from MCNPX-2.6, PHITS and TALYS-1.0 for these activation-relevant reactions. Fig. 6 for the reaction ${ }^{56} \mathrm{Fe}$ $(\mathrm{d}, \alpha)^{54} \mathrm{Mn}$ illustrates the different behaviors. This shows: (i) the poor behavior of MCNPX and PHITS, (ii) that the TALYS (with globa] parameters) results follow the shape of the experimental data [13]

Table 1

Neutron yield per $9 \mathrm{MeV}$ incident deuteron on natural copper and iron.

\begin{tabular}{lllll}
\hline & $\begin{array}{l}\text { ISABELfDresner/ } \\
\text { RAL }\end{array}$ & INCL4/ABLA & TALYS-1.0 & PHITS \\
\hline${ }^{63} \mathrm{Cu}$ & $1.66 \times 10^{-4}$ & $2.22 \times 10^{-4}$ & $5.66 \times 10^{-4}$ & $4.96 \times 10^{-4}$ \\
$(69.17 \%)$ & $\left(1.14 \times 10^{-4}\right)$ & $\left(1.53 \times 10^{-4}\right)$ & $\left(3.91 \times 10^{-4}\right)$ & \\
${ }^{65} \mathrm{Cu}$ & $1.83 \times 10^{-4}$ & $2.71 \times 10^{-4}$ & $1.00 \times 10^{-3}$ & \\
$(30.17 \%)$ & $\left(5.64 \times 10^{-5}\right)$ & $\left(8.17 \times 10^{-5}\right)$ & $\left(3.08 \times 10^{-4}\right)$ & $7.26 \times 10^{-4}$ \\
Cu nat. & $1.71 \times 10^{-4}$ & $2.37 \times 10^{-4}$ & $7.00 \times 10^{-4}$ & $5.80 \times 10^{-4}$ \\
Fe nat. & $9.73 \times 10^{-6}$ & $1.14 \times 10^{-5}$ & $4.40 \times 10^{-4}$ & - \\
\hline
\end{tabular}

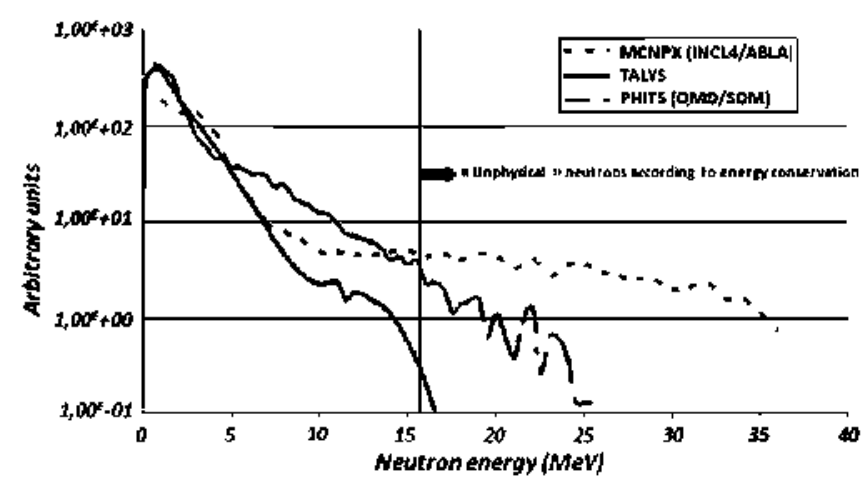

Fig. 5. Energy spectrum of emitted neutron from natural copper for $9 \mathrm{MeV}$ incident deuterons. 


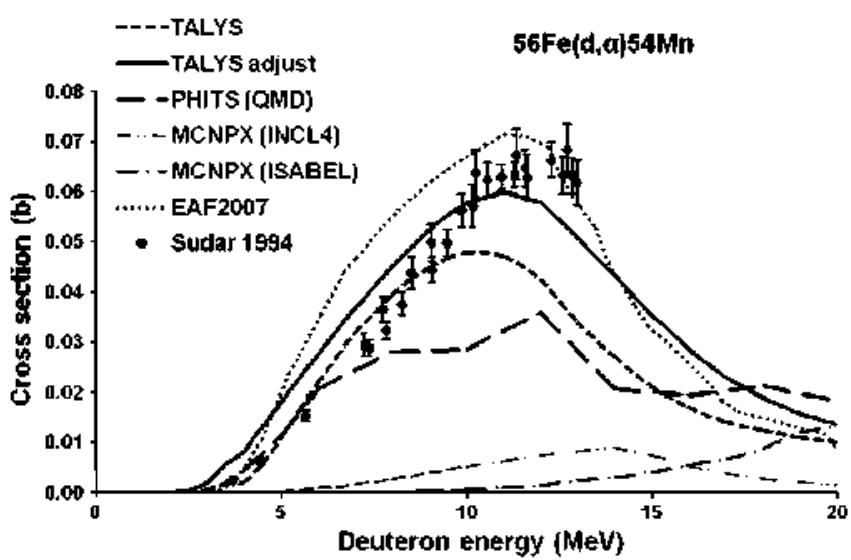

Fig. 6. Cross section for ${ }^{56} \mathrm{Fe}(\mathrm{d}, \propto)^{54} \mathrm{Mn}$ reaction: comparison of evaluated and measured data [13].

but do not fit the amplitude, and (iii) that when TALYS is used with parameters obtained from the mathematical fitting calculations mentioned in Section 3, the results reproduce well the experimental cross sections. It is important to stress the development effort performed within the fusion community leading to the deuteroninduced activation data library included in EAF-2007. This library, which includes 66,864 reactions, is mainly based on model calculations with a previous version of the TALYS code (using global parameters). Only a limited number of reactions had been obtained using TALYS, but with local parameters [7], and a few renormalizations to EXFOR data have been made. The reaction considered in Fig. 6 is one of the limited number for which fitting has been applied in the data included in EAF. As can be seen, EAF values are close to those obtained with the current TALYS version using the parameters derived from our fitting calculations.

\section{Conclusions}

The benchmark performed has shown that:

1. None of the models used to calculate deuteron cross sections of the nuclei studied using either a nuclear model dedicated code such as TALYS or Monte Carlo codes such as MCNPX and PHITS with built-in analytical models gives a good fit to experimental values. In addition. MCNPX and PHITS are inappropriate because they generate nonphysical high energy tail neutrons.

2. Even if results obtained with TALYS (with default-global parameters) are not better in amplitude than those obtained with MCNPX and PHITS, they give a shape that fits rather well the shape of experimental values.
3. The utilization of TALYS with appropriate adjusting parameters allows a good reproduction of experimental cross sections for safety and radioprotection calculations.

This work will be extended for the IFMIF facility with energies up to $40 \mathrm{MeV}$ and with additional materials relevant to JFMIF.

Consequently, a good option for addressing transport simulations and prompt dose calculations in EVEDA is the development of a computational procedure based on extending Monte Carlo codes to use data files generated by the TALYS with appropriate adjusting parameters. An extended version of MCNPX, called MCUNED, is already implemented and is under testing. Regarding activation and residual dose rate calculations, in addition to selecting an appropriate activation code, such as FISPACT [14] or ACAB [15], a good option can be to use the deuteron activation file EAF-2007 (and later updated versions) and when necessary TALYS-generated activation data with appropriate adjusting parameters.

\section{Acknowledgements}

The present work has been carried out in the frame of the Fusion broader approach between Japan and Europe. It is part of the contribution of the French and Spanish Home Teams to the IFMIF/EVEDA project within the accelerator system group (ASG) of the European team. On the Spanish side, it has been partially supported by Plan Nacional de [ + D + ] (2008-2011) Fusion Nuclear. ENE2008-06403-C06-02, MICINN, Spain.

\section{References}

|1] P. Garin, M. Sugimoto, Fusion Eng. Des. 84 (2009) 259.

[2] D.B. Pelowitz, ed., MCNPX: user's manual, v2.6.0, LA-CP-07-1473, April 2008. <http:/fmenpx.lanl.govs.

|3| K. Niita, T. Sato, H. Iwase, H. Nose, H. Nakashima, l. Sihver, Radiat. Meas, 41 (2006) $1080-1090$

|4| J. Sanz, M. Galcía, F. Ogando, A. Mayoral, D. López, P. Sauvan, B. Brañas, Fusion Sci. Technol. 56 (1) (2009) 273-280.

[5] A.]. Koning, S. Hilaire, M.C. Duijvestijn, TALYS-1.0, in: O. Bersillon, F. Gunsing, E. Bauge. R. Jacqmin, 5. leray (Eds.). Proceedings of the lnternational Conference on Nuclear Data for Science and Technology (ND2007), EDP Sciences, Nice, 2008, pp. 211-214

[6] R.A. Forrest, ]. Kopecky, J,-CH. Sublet, The European activation file: EAF-2007 deuteron and proton-induced cross section libraries, UKAEA FUS-536, 2007.

17| A. Hermanne et al., Nucl. Instrum. Methods Phys. Res., Sect. B 258 (2007) 308.

[8] M. Avrigeanu, V. Avrigeanu, F.L. Roman, Deuteron-induced activation cross section evaluation for accelerator materials. Progress Report to the EURATOMMEdC Association for the time period 2006-01-01 to 2006-06-30. Task: TW5TTMl-004, Deliverable No. 6, December 2006 (restricted to the working group)

|이 P. Bem et al., Conf, Nucl. Data Sci. Technol., Nice, 2007.

[10] H. Okamura et al., Nucl. Phys., Sect. A 169 (1971) 401.

[11] F.W. Pement, R.L. Wolke, Nucl. Pliys. 86 (1966) 429.

[12] T. Zhenlan et al., At. Energy Sci. Technol, 18 (1984) 506.

[13] S. Sudar, S.M. Qaim, Phys. Rev. C 50 (1994) 2408.

[14] R.A. Forrest, FISPACT-2007: user's manual, UKAEA FU5-534, 2007

[15] J. Sanz et al., ACAB: inventory code for nuclear applications, User's manual V2008, NEA-1839, 2009. 